\title{
Flat polyps depress gastroenterologists
}

\author{
Stephen B Hanauer
}

Two articles published in March 2008 reflect a 'see [sic] change' for the way in which gastroenterologists detect colorectal neoplasia (CRN). Soetikno and colleagues described the prevalence of nonpolypoid (flat and depressed) CRN in asymptomatic and symptomatic adults (JAMA [2008] 299: 1027-1035), and the updated joint guidelines from the American Cancer Society, the US Multisociety Task Force on Colorectal Cancer, and the American College of Radiology considered screening and surveillance for the detection of early colorectal cancer and adenomatous polyps (CA Cancer J Clin [2008] doi:10.3322/ CA.2007.0018). The findings of these articles provide both challenges and opportunities for gastroenterologists who aim to optimize their use of the rapidly evolving technologies available for the detection of CRN.

While the American College of Gastroenterology has consistently proponed optical colonoscopy as the preferred screening and surveillance strategy to detect $\mathrm{CRN}$, the radiology community has continued to recommend barium enemas and, most recently, virtual or CT colonography as an alternative strategy, with the support of the American Cancer Society and the US Multisociety Task Force on Colorectal Cancer. The expansion of colonoscopic screening (since Medicare became willing to pay for it) has significantly increased the productivity and entrepreneurial efforts of gastroenterologists. Consequently, these efforts are subject to increasing scrutiny from numerous sectors including the US Congress (in relation to patients' self-referrals), third-party payers and the legal profession, and their involvement has led to increasing demand for the introduction of quality screening initiatives. Already, guidelines are being established on 'withdrawal times' and the 'percentage of positive screening examinations' for use in polyp identification.

In the field of IBD, our ability to detect CRN in the setting of ulcerative colitis has increased

\section{...we can \\ anticipate \\ that gastro- \\ enterologists \\ will work \\ increasingly \\ hard to find the \\ least-visible \\ CRN lesions...}

SB Hanauer is Editorin-Chief of Nature Clinical Practice Gastroenterology \& Hepatology.

\section{Competing interests}

The author declared no competing interests.

www.nature.com/clinicalpractice doi:10.1038/ncpgasthep1149 considerably. We have moved from the concept that dysplasia could develop in 'flat mucosa' to become dysplasia associated lesions or masses, identified with fiberoptic scopes, to the recognition of more subtle mucosal lesions that harbor dysplasia, detected with highresolution videoscopes (with or without chromoendoscopy). At the same time, our Japanese colleagues, who are usually several years ahead of those in the West when it comes to use of high-resolution techniques, have similarly recognized lesions in otherwise healthy colons, which despite their 'nonpolypoid' appearance, are more likely to harbor carcinoma than typical, raised polyps of the same diameter. We frankly do not know what the ability of CT colonography is to identify these flat lesions that have increased malignant potential.

Nonetheless, the era of production-line screening colonoscopy is coming to an end. Take note that, before embarking on their study, Soetikno and colleagues “...developed a gastroenterology and pathology faculty exchange program with leading Japanese endoscopy centers..." to learn how to identify the characteristic features of nonpolypoid CRN. This type of learning will need to be incorporated into current Gl training curricula, and previously trained colonoscopists will need to update their skills. Already in place is the expectation and documentation of a minimum withdrawal time that averages $6 \mathrm{~min}$. For the investigators in Soetikno and colleagues' study, the average procedure time needed to identify the $\sim 6 \%$ of lesions that were nonpolypoid $\mathrm{CRN}$ in the screening population was $33 \mathrm{~min}$.

Screening for $\mathrm{CRN}$ is already effective and will become even more effective as technologies continue to evolve. However, we can anticipate that gastroenterologists will work increasingly hard to find the least-visible CRN lesions and, at the same time, performing more therapeutic procedures to remove lesions identified by nonoptical examination. 